\title{
Small RNA profiling of Cavendish banana roots inoculated with Fusarium oxysporum f. sp. cubense race 1 and tropical race 4
}

Shulang Fei ${ }^{1}$, Elizabeth Czislowski ${ }^{2}$, Stephen Fletcher ${ }^{1}$, Jonathan Peters ${ }^{1}$, Jacqueline Batley ${ }^{2,3}$, Elizabeth Aitken ${ }^{2}$ and Neena Mitter ${ }^{1 *}$

\begin{abstract}
Fusarium wilt, caused by the soil-borne fungal pathogen, Fusarium oxysporum f. sp. cubense (Foc), is considered as one of the most threatening diseases of banana. The Cavendish variety, resistant to Foc race 1 (R1), is susceptible to tropical race 4 (TR4), an aggressive race of the pathogen which is of increasing concern worldwide. Previous studies have revealed that plant small RNAs (sRNAs) play crucial roles in the host response to pathogen infection. To investigate the roles of sRNAs involved in the interaction of the banana-Foc pathosystem, small RNA profiles of Cavendish banana roots inoculated with Foc TR4 and Foc R1 were obtained and analyzed in the present study using Next-Generation Sequencing (NGS) technology. A total of 112 discrete mature microRNAs (miRNAs) belonging to 22 known miRNA families were found across all constructed sRNA libraries. The expression of miR166, miR159 and miR156 was upregulated in TR4-inoculated samples as compared to mock-inoculated samples, while the expression of these miRNAs was approximately the same in R1-inoculated and mock-inoculated samples. Consistent with the sequencing data, qRT-PCR results demonstrated up-regulation of these miRNAs and down-regulation of their target genes in TR4-inoculated samples, but not in R1-inoculated samples. Considering Cavendish banana is resistant to R1 and susceptible to TR4, it is possible that these sRNAs and their target genes are involved in particular plant defence pathways such as salicylic acid-based defence. The findings will pave way for future investigations of the defence mechanism and potential approaches of resistance improvement.
\end{abstract}

Keywords: Banana, Foc, R1, TR4, Inoculation, Small RNA, miR156, miR159, miR166

\section{Background}

Fusarium wilt is a devastating disease of banana, caused by the fungal soil-borne pathogen, Fusarium oxysporum f. sp. cubense (Foc). It infects the banana plants from the roots, then travels up and blocks the transport of water and nutrients, causing plant death (Pietro et al. 2003; Ploetz 2015b). The outbreak of Foc race 1 (R1) almost destroyed the banana cultivar Gros Mitchel, which was almost the sole banana cultivar traded worldwide in the 1900s (Ploetz 2015b). Even though the alternative option, Cavendish, is resistant to $F o c$ R1, a relatively new Foc race, tropical race 4 (TR4) is pathogenic on all available commercially produced banana cultivars, including Cavendish.

\footnotetext{
*Correspondence: n.mitter@uq.edu.au

'Queensland Alliance for Agriculture and Food Innovation, The University of Queensland, St Lucia, QLD 4072, Australia

Full list of author information is available at the end of the article
}

The longevity of $F$. oxysporum in soil is very high and is confounded by the lack of effective control methods (Hwang and Ko 2004; Ploetz 2006; Dale et al. 2017). In order to develop potential novel solutions for management of Foc, a greater understanding of the molecular mechanisms underlying the interaction between the pathogen and the host needs to be explored.

Small RNAs (sRNAs) are short non-coding RNA molecules of 20-26 nucleotides (nt) that regulate a range of eukaryotic biological processes and play crucial roles in responding to biotic and abiotic stresses in plants. Two major classes of sRNAs in plants are microRNAs (miRNAs) and small interfering RNAs (siRNAs). The class miRNAs are derived from endogenous genes and processed from single-stranded hairpin RNA (hpRNA), which can form a stem-loop secondary structure. With regards to the siRNAs, they generally originate from double-stranded RNA

(c) The Author(s). 2019 Open Access This article is distributed under the terms of the Creative Commons Attribution 4.0 International License (http://creativecommons.org/licenses/by/4.0/), which permits unrestricted use, distribution, and reproduction in any medium, provided you give appropriate credit to the original author(s) and the source, provide a link to the Creative Commons license, and indicate if changes were made. The Creative Commons Public Domain Dedication waiver (http://creativecommons.org/publicdomain/zero/1.0/) applies to the data made available in this article, unless otherwise stated. 
(dsRNA) either from exogenous viruses or from endogenous repetitive regions or other complementary transcripts (Axtell 2013; Kamthan et al. 2015). The intermediate dsRNA or hpRNA is then cleaved by Dicer-Like (DCL) endonucleases into 20-26 base-pair (bp) double-stranded duplexes, one strand of which is loaded into Argonaute (AGO) proteins and forms the RNA-Induced Silencing Complex (RISC). Then the RNAi mechanism is achieved by either the post-transcriptional silencing (PTGS) or transcriptional silencing (TGS) pathways. In PTGS, the RISC leads the miRNA or siRNA to its complementary sequences of the targeted mRNAs and represses expression by slicing the targeted mRNAs. In general, the action of siRNAs leads to cleavage of mRNAs with perfectly matching sequences; whereas miRNAs target transcripts that are not fully complementary. In TGS, the 24 nt siRNAs bind to genomic DNA and inhibit gene transcription by DNA methylation and chromatin remodeling (Vaucheret 2006; Castel and Martienssen 2013).

Numerous studies have demonstrated that plant sRNAs are actively involved in the defence response upon pathogen attack (Ruiz-Ferrer and Voinnet 2009; Sheeba et al. 2013; Gupta et al. 2014; Islam et al. 2017). Up-regulation of miR393 was observed following infection by Pseudomonas syringae in Arabidopsis thaliana, which consequently repressed the expression of auxin receptor genes so that growth of the pathogenic $P$. syringae was inhibited (Navarro et al. 2006). Research by Fahlgren et al. (2007) confirmed this phenomenon. They also demonstrated miR160 and miR167 were up-regulated after $P$. syringae infection, suppressing the expression of transcription factors of the ADP-ribosylation factor (ARF) family, which are key factors of auxin signaling. Overexpression of miR396a-5p was found to increase the susceptibility to Phytophthora nicotianae infection in tobacco (Chen et al. 2015), suggesting miR396a-5p plays a crucial role in biotic stress by targeting growth-regulating factors (GRFs). An early example of sRNAs involved in host-fungi interaction was in loblolly pine challenged by the rust fungus Cronartium quercuum f. sp. fusiforme (Lu et al. 2007). In the loblolly pine-rust system, ten miRNA families such as miR156, miR159 and miR160 were found to be significantly down-regulated during the infection. The expression of their target transcripts, ARF10, SQUAMOSAPROMOTER BINDING PROTEIN (SBP), MYELOBLASTOSIS (MYB) and Aux/IAA protein genes, was correspondingly enhanced to defend against the infection. In tomato infected with Fusarium oxysporum, two miRNAs, miR482f and miR5300, targeting NB-LRR immune genes were repressed in resistant cultivars so that their target genes were activated and able to play their roles in the plant immune response (Ouyang et al. 2014). In addition to the endogenous sRNAs functioning in the host, miR166 and miR159 in cotton were found to be exported into the fungal pathogen Verticillium dahliae (Zhang et al. 2016), which inhibited the expression of two fungal virulence genes and increased disease resistance. In terms of the banana-Foc pathosystem, studies on banana sRNA identification have emerged in recent years (Ghag et al. 2015; Song et al. 2015; Song et al. 2016), however little is known concerning the roles of banana sRNAs in response to Foc invasion. In a recent report, members of the miR169 family showed higher expression level in resistant banana cultivars upon Foc inoculation than in susceptible cultivars, suggesting their involvement in Foc resistance in banana (Song et al. 2018).

In this study, we applied deep sequencing technology to obtain comparative sRNA profiles of Cavendish banana roots inoculated with Foc R1 and Foc TR4, aiming to identify the sRNAs that were differentially regulated by $\mathrm{R} 1$ and TR4 and thus gain more knowledge of the molecular mechanisms of the host's defence response during pathogen invasion.

\section{Results}

An overview of the sequencing data

At 28 days post inoculation (dpi), banana root tissues were harvested for total RNA extraction at the two inoculation locations. Twelve small RNA libraries were constructed from four treatment groups, namely the TR4_C (TR4 mock-inoculated), TR4_I (TR4-inoculated) both from the Northern Territory (NT) site and the R1_ C (R1 mock-inoculated), and R1 I (R1-inoculated) from the Brisbane site. In all cases three biological replicates were used for each treatment. From the twelve sRNA libraries, a total of $12.21 \mathrm{~Gb}$ of raw data was generated. Following adapter removal and high-quality read screening, an average of 2,869,477, 3,438,027, 2,112,927 and 2, 262,496 processed reads from three replicates were obtained from TR4_C, TR4_I, R1_C and R1_I groups, respectively.

\section{Identification of known miRNAs in response to FoC infection}

To investigate the regulatory response of known conserved miRNAs to Foc infection, the MiRDeep2 package was used to align the processed reads from all four groups to the miRNA database, miRBase. A total of 112 miRNAs belonging to 22 known miRNA families were found with at least one raw read from the sRNA libraries mapping to the database. More than ten members from each of the miR156, miR166, miR167, miR395 and miR396 families were found in the twelve sRNA libraries, while fewer members from other miRNA families were present in these sRNA libraries (Fig. 1). With regard to the overall expression of different miRNA families (Fig. 2), miR166, miR396 and miR159 had the highest abundance regardless of their differential expression in each treatment group. 


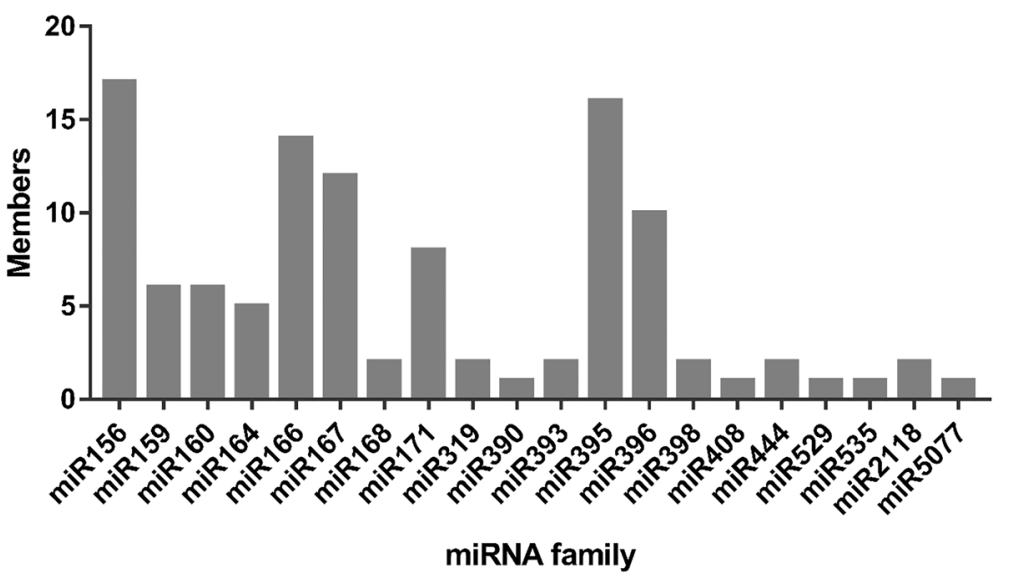

Fig. 1 Number of miRNA members belonging to the miRNA families found in this study. The miRNA families listed on the X-axis are the identified miRNA families in the twelve sRNA libraries. Among them, miR156, miR395, miR166 and miR167 possessed the most members present in the sRNA libraries

Whereas miR398, miR529, miR444, miR2118, miR394, miR393 and miR5077 were only found in TR4 libraries but not in R1 libraries.

To investigate the role of miRNAs in root responses to Foc inoculation, differential expression analysis was performed for miRNAs which had at least one normalized read (RPMR, reads per million reads) in each sRNA library (Fig. 3). Fold change of TR4_I versus TR4_C and R1_I versus R1_C is shown in Additional file 1: Figure S1. Direct comparison of miRNA expression of TR4 groups with R1 groups was not conducted due to trials being at different locations. However, comparison patterns of the inoculated group versus control group in both the TR4 and R1 groups were obtained. Different expression patterns of the TR4 groups and R1 groups were observed. Overall, less difference was observed between R1_I and
R1_C than between TR4_I and TR4_C. The expression of miR156a, miR159f and miR166c in TR4_I was 5, 3.9 and 2.9 times higher than in TR4_C, while in the R1 samples, the fold change for these same miRNAs between R1_I and R1_C was lower (Additional file 1: Figure S1). In addition to the above miRNAs with different expression patterns in response to TR4 and R1 inoculation, miR396 expression was reduced in TR4 inoculated samples, while miR408 expression was reduced in both TR4 and R1 inoculated samples.

\section{Identification of novel miRNAs in four SRNA libraries}

To predict novel miRNAs present in the twelve sRNA libraries, processed reads were mapped to the banana genome sequence (http://banana-genome-hub.southgreen.fr/ download) using the MiRDeep2 package. Reads that mapped to the miRBase and Rfam databases were removed

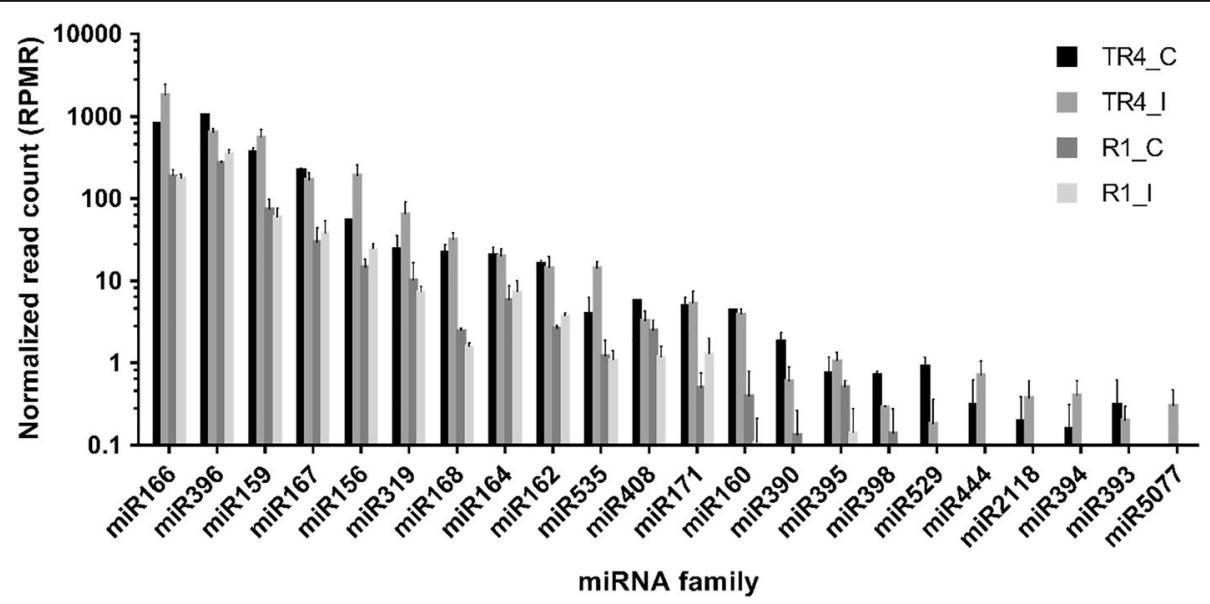

Fig. 2 MiR166, miR396 and miR159 possessed the highest expression in the treatment groups. The miRNA families listed on the X-axis are the identified miRNA families in the twelve sRNA libraries. The normalized read count on the Y-axis was calculated as "reads per million reads" (RPMR) for each sRNA library. The logarithmic scale was used to display the normalized read count. Error Bars represent Standard Error of the Mean (SEM) 


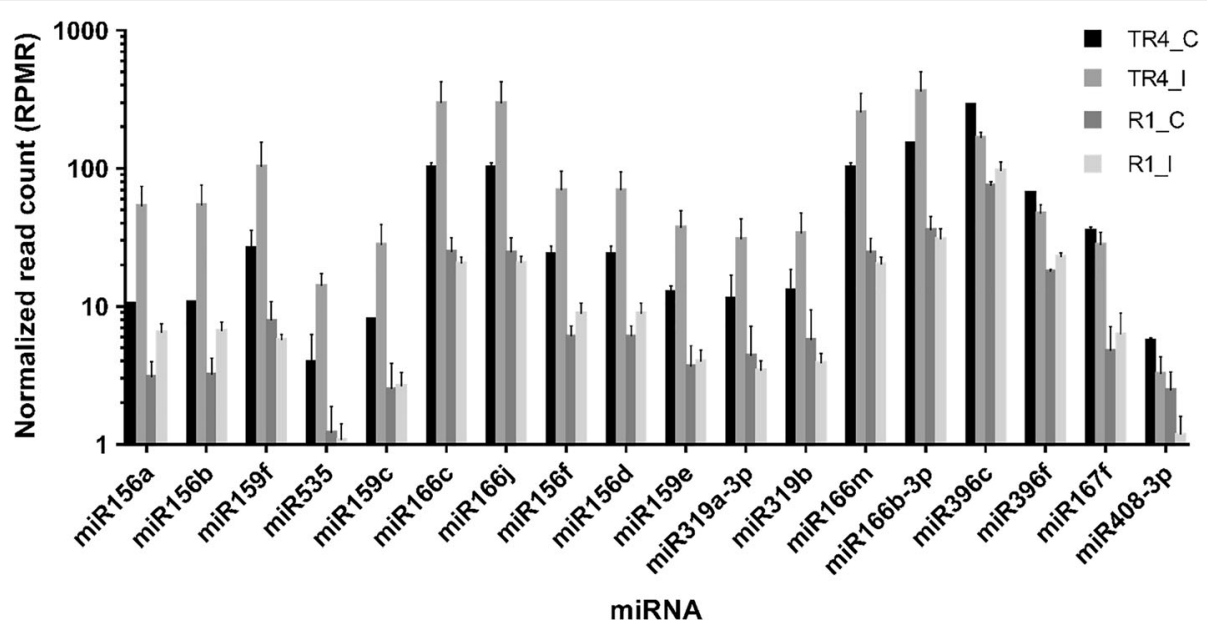

Fig. 3 Expression of miRNAs in each treatment group. Members of miR156, miR159 and miR166 families showed increased expression in TR4_I as compared to TR4_C. Not as much increase was induced by R1 inoculation. The normalized read count on the Y-axis was calculated as "reads per million reads" for each sRNA library. The logarithmic scale was used to display the normalized read count. Error Bars represent Standard Error of the Mean (SEM)

(Griffiths-Jones et al. 2008). The folding free energy of the predicted miRNA precursors was determined and those with randfold $P$-value higher than 0.05 were removed (Bonnet et al. 2004). As a result, 29 putative novel miRNAs were obtained (Additional file 2: Table S1).

\section{MiRNA target gene prediction and GO analysis}

Target genes of the putative known and novel miRNAs were predicted using the online tool psRNATarget. With a maximum expectation score of 3.5, 279 and 365 target genes were predicted for the putative known miRNAs and novel miRNAs found above, respectively (Additional file 3: Table S2). To further understand their function, gene ontology (GO) analysis of the
miRNA target genes was performed using Blast2Go. Overall, seven putative biological processes, three molecular functions and six cellular components were predicted for the putative target genes of the identified known miRNAs (Fig. 4). Binding and cell part comprised the most genes in molecular function category and cellular component category respectively. Regulation of transcription showed the highest percentage in biological processes (Fig. 5a), suggesting a high proportion of these target genes were involved in regulating gene expression during the pathogen invasion. GO analysis was also performed for predicted target genes of the putative novel miRNAs. Seven biological processes, three molecular functions and seven cellular components were predicted for the novel miRNA

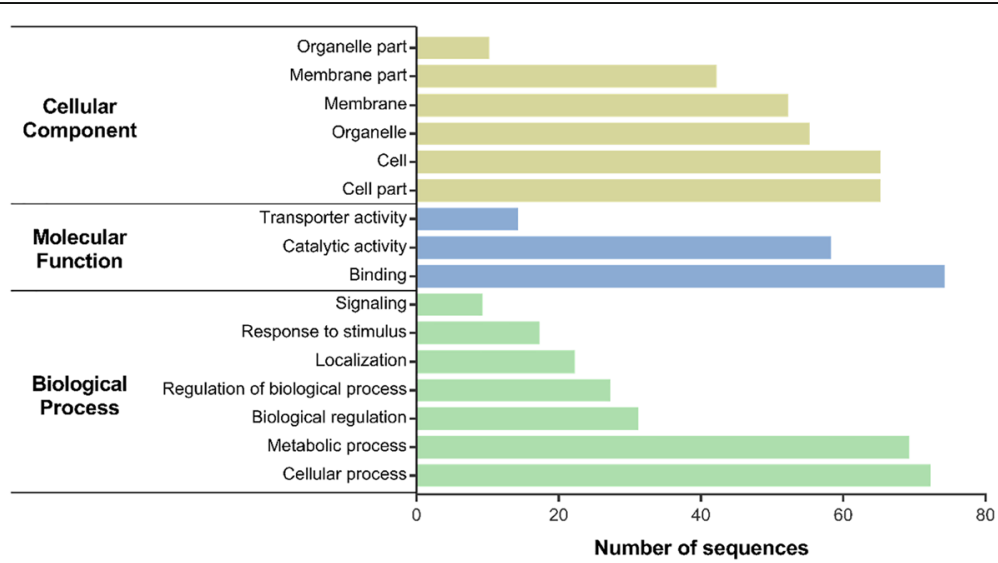

Fig. 4 Gene ontology analysis of putative target genes of the identified known miRNAs. A total of six terms in the cellular component category, three terms in the molecular function category and seven terms in the biological process category were obtained. "Binding" and "Cellular process" possessed the most genes among all the GO terms found in this study 


\section{a}
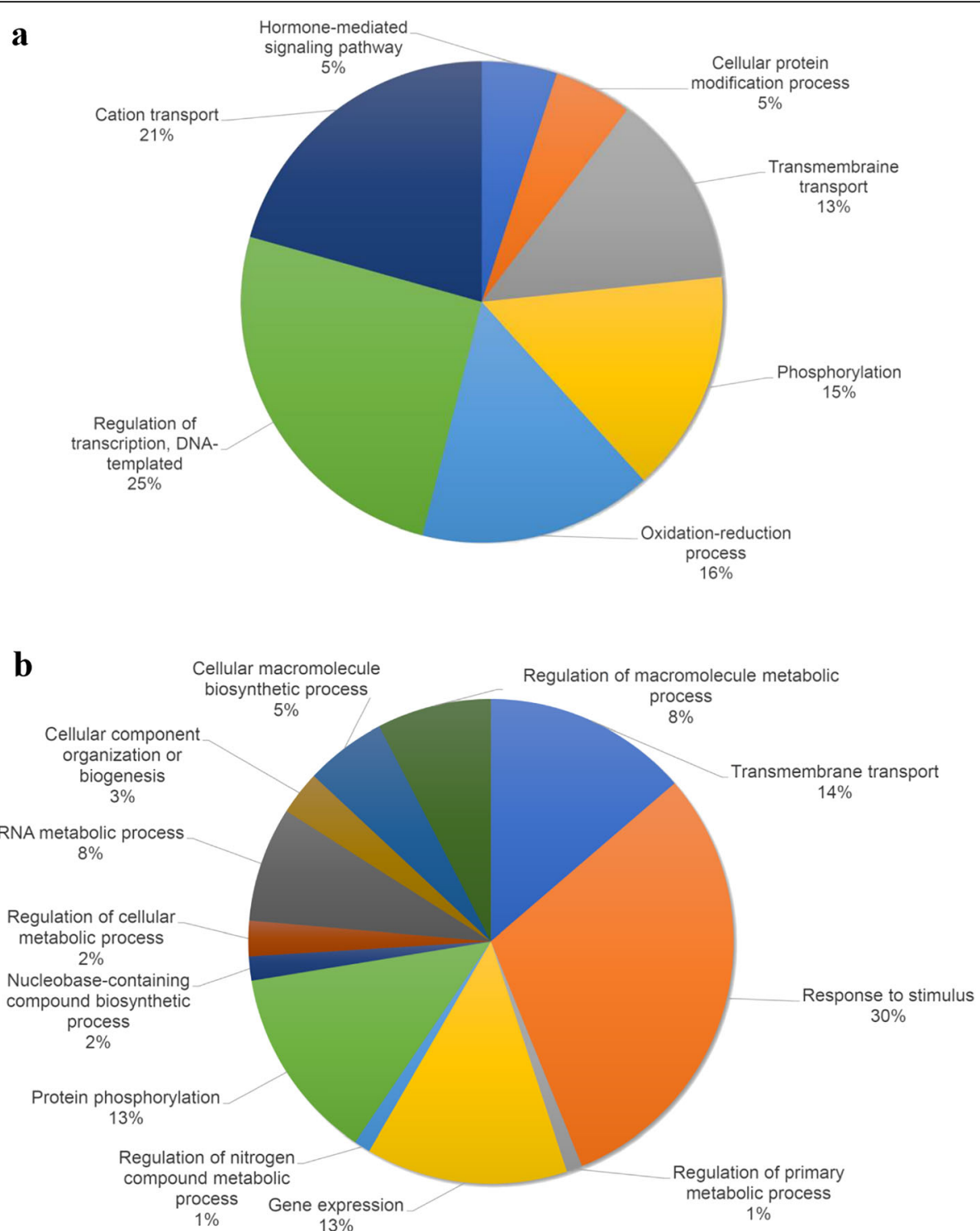

Fig. 5 Distribution of the predicted target genes in gene ontology terms of the biological process category. a Predicted target genes of the identified known miRNAs. b Predicted target genes of the putative novel miRNAs. "Regulation of transcription" and "Response to stimulus" comprised the most abundant target genes of the identified known miRNAs and putative novel miRNAs, respectively

target genes. In the gene distribution of biological processes, "response to stimulus" accounted for $30 \%$ (Fig. 5b), which indicated that these miRNAs and their targeted genes could be actively involved in the host response to F. oxysporum.

\section{qRT-PCR validation of miRNAs and target genes}

Quantitative real-time PCR (qRT-PCR) was conducted to validate the expression level of the miRNAs and their target genes. Three miRNAs of the most interest, miR159, miR156 and miR166, were chosen for validation. For miRNA qRT-PCR, miR399 was used as the reference miRNA for normalization due to its constant expression level in different banana tissues, as well as under stress conditions (Muthusamy et al. 2015). L2 was used as the reference gene for target gene expression normalization (Podevin et al. 2012).

As shown in Fig. 6, miR166, miR159 and miR156 showed higher expression in TR4_I than in TR4_C. In contrast, expression of their predicted target genes, HOX9, GAMYB and SPL12 was significantly reduced in TR4_I as compared to in TR4_C. This negative correlation of expression between miRNAs and target genes is consistent with the sequencing data (Fig. 3). Thus, the up-regulation of these miRNAs upon TR4 
a

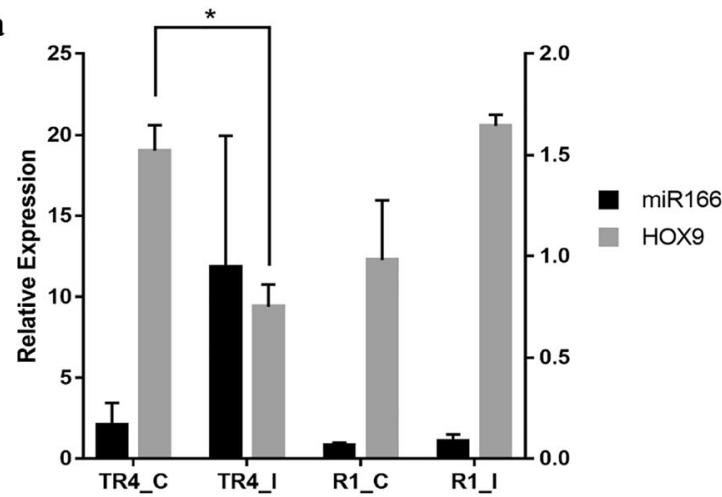

b
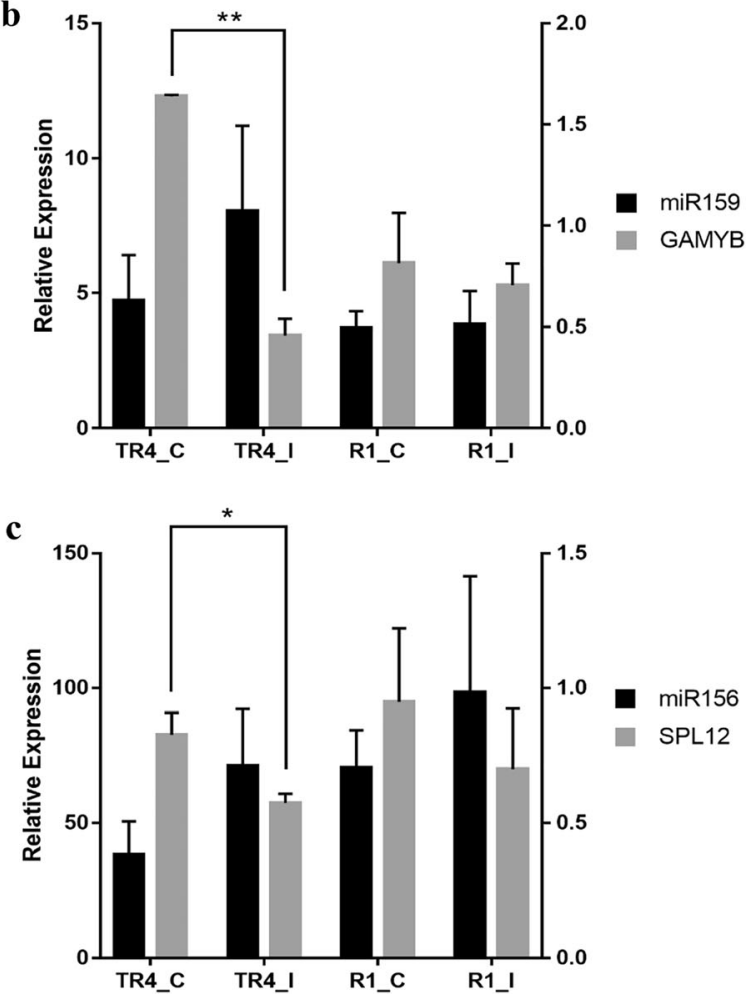

Fig. 6 qRT-PCR validation of selected miRNAs and their target genes. Negative-correlation of the expression between miR166, miR159, miR156 and their target genes was observed in TR4_C and TR4_I. a miR166 and its target gene, HOX9 (Homeobox-leucine zipper protein 9) (GSMUA_Achr5T18530_001). b miR159 and its target gene, GAMYB (GSMUA_Achr7T21560_001). c miR156 and its target gene, SPL12 (Squamosa Promoter Binding Protein-like 12) (GSMUA_Achr10T02970_001). One-way analysis of variation (ANOVA) was used to determine the significant difference of the target gene expression between groups. One asterisk represents significant difference with $P$-value $<0.05$; two asterisks represent significant difference with $P$-value $<0.01$

inoculation caused downstream down-regulation of their target genes, suggesting the involvement of these genes during the interaction.

Exploration of banana siRNAs in response to Foc infection To investigate whether any banana endogenous siRNAs played roles in responding to Foc invasion, the processed reads of the sRNA libraries were mapped to the banana genome sequence (with intron and UTRs). The abundance of $21 \mathrm{nt}, 22 \mathrm{nt}$ and $24 \mathrm{nt}$ perfectly mapped reads was then compared between inoculated and mockinoculated samples using the SCRAM program (Fletcher et al. 2018). Figure 7 is a list of representative banana genes with various $21 \mathrm{nt}$ and $24 \mathrm{nt}$ mapped read abundance in the four treatment groups (Additional file 4: Table S3). Ma02_g06960 had the highest abundance of 21 nt mapped reads and both TR4 and R1 inoculated samples had approximately doubled mapped reads as compared to control samples (Additional file 1: Figure S2). Ma08_ g19220 also showed a similar increase pattern in TR4- 


\section{a 21nt}

$\mathrm{Ma} 02$ g06960 Uncharacterized protein

Ma05_g17090 Uncharacterized protein

Mall_gl 5080 Probable alkaline/ncutral invertase B

Ma06_g23000 Hypothetical protein

Ma08_g19220 Uncharacterized protein

Ma06_g18190 nuclcotidyltransferase, putative, expressed

Ma08_g18420 Putative Tropinone reductase homolog At2g29150

Ma05_g05580 Uncharacterized protein

Ma05_g05570 Uncharacterized protcin

$\mathrm{Ma06}$ g02840 region found in RelA/SpoT proteins containing protein, expressed

Ma04_g10650 1,2-dihydroxy-3-keto-5-methylthiopentene dioxygenase 1

Ma06_g22630 Histone-lysine N-methyltransferase EZ1

Ma07_g22960 Putative Wall-associated receptor kinase 5

Ma07_gl4160 Uncharacterized protein

mito3_g00140

Ma04_g2 1360 Probable copper-transporting ATPase HMA5

Ma08_g09870 Putative Probable esterase PIR7A

Ma03_g28620 Uncharacterized protein

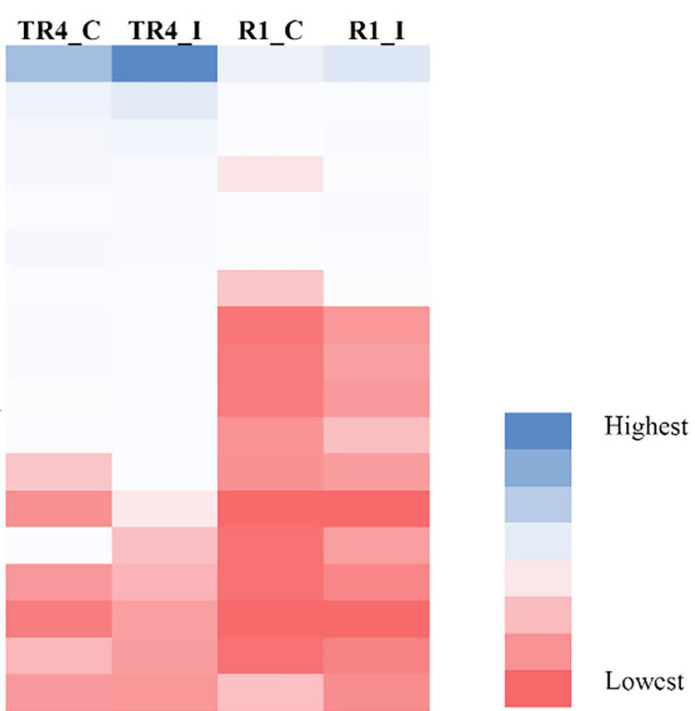

b 24nt

Ma00_g03310 Uncharacterized protein

Mal1_gl5080 Probable alkaline/neutral invertase B

Mall_g14010 Ribulose-phosphate 3-epimerase, cytoplasmic isoform

Ma00_g03320 Uncharactcrized protcin

Ma00_g03290 Uncharacterized protein (multiple hotspot location)

Ma11_g20390 nicotiana lesion-inducing like, putative, expressed

Mal0_g20970 Uncharacterized protein

Ma05_g15620 Cellulose synthase A catalytic subunit 7 [UDP-forming]

Ma10_g01100 Sodium/hydrogen exchanger 2

Ma08_gl 6280 protein kinase domain containing protcin, cxpressed

Ma04_g33910 Putative S-norcoclaurine synthase 2

Ma09_g05270 S-type anion channel SLAH3

Mal0_g27040 Protein arginine N-methyltransferase PRMT10

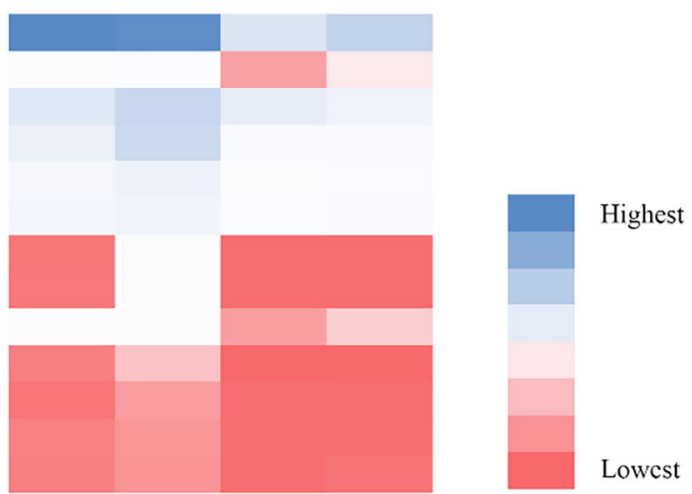

Fig. 7 Increased abundance of the reads mapping to some banana genes was observed in inoculated samples as compared to control. a $21 \mathrm{nt}$ mapped reads. b $24 \mathrm{nt}$ mapped reads. Banana genes with annotated protein name were listed in the left column. The four columns on the right represent the abundance of mapped reads from respective treatment group. The darkest blue color represents the highest mapped read count, and the darkest pink color represents the lowest mapped read count. Exact read count is listed in Additional file 4: Table S3

inoculated and R1-inoculated samples as compared to TR4-control and R1-control samples respectively. However, both genes encode uncharacterized proteins. For the $24 \mathrm{nt}$ sRNAs, the abundance of reads mapped to Ma11 g15080, encoding alkaline/neutral invertase B, was increased upon both TR4 and R1 inoculation, with R1 inoculation causing a more significant increase.

Some genes showed different comparative patterns between R1 groups and TR4 groups, which might provide an insight into how Cavendish responded differently to the two Foc races. Among these genes, Ma06_g22630 (Histonelysine N-methyltransferase EZ1) and Ma07_g22960 (Putative wall-associated receptor kinase 5) showed 1.6-fold and 1.7 -fold increase, respectively, of $21 \mathrm{nt}$ mapped reads in TR4-inoculated samples than control samples, while no difference was observed between R1-inoculated and control samples. Similarly, 24 nt sRNA abundance of Ma00_g03320
(Uncharacterized protein), Ma08_g16280 (Protein kinase domain containing protein, expressed) and Ma04_g33910 (Putative S-norcoclaurine synthase 2) showed an increase of 2.6 times, 3.7 times and 3.5 times in TR4-inoculated samples over the control samples respectively, whereas the sRNA abundance in R1-inoculated samples and R1-control samples were parallel. Ma10_g20970 (Uncharacterized protein) showed 12-fold increase in TR4_I samples than TR4 C samples, while less than one mapped RPMR was found in R1_I and R1_C. The mapping profiles of these genes showed only one or two hotspots of the antisense-mapped sRNAs. Instead of being randomly distributed along the whole gene, which could be caused by degradation, it was likely that these sRNAs were playing roles in response to the pathogen inoculation. Apart from Ma10_g20970 encoding an uncharacterized protein, the sRNA mapping hotspots were mostly located in the intron regions of the genes 
of interest and mapped to the antisense strand. It is possible that the abundant $21 \mathrm{nt}$ sRNAs were intron-derived siRNAs that can bind to their host DNA sequence and cause DNA methylation in cis manner (Chen et al. 2011), and the $24 \mathrm{nt}$ sRNAs were hc-siRNAs (heterochromatin-siRNAs). Further work is required to confirm this speculation.

\section{Discussion}

Small RNAs are important regulators of plant development and the plant's immunity system. Since the genome sequence of banana has been available, studies on banana miRNAs have been accumulating (D'Hont et al. 2012; Davey et al. 2013; Chai et al. 2015; Ghag et al. 2015). A number of banana miRNA families have been identified. MiRNAs responding to drought stress and salt stress were investigated by Muthusamy et al. (2015) and Lee et al. (2015). However, research on banana miRNAs in response to biotic stress remains limited. This study investigated the small RNA profile of Cavendish banana inoculated with the Foc R1 and TR4 with an attempt to understand the roles of the small RNA regulators in banana upon Foc invasion.

By means of high throughput sequencing technology, this study has identified 112 previously-identified miRNAs belonging to 22 miRNA families from the sRNA libraries of both R1-inoculated and TR4-inoculated banana roots. Consistent with a previous finding (Ghag et al. 2015), miR166 showed the highest abundance among all the known miRNAs in banana. By challenging the plants with $F o c$, we found the miRNA profiles were altered by the inoculation of Foc TR4, whereas not as much change occurred during Foc R1 inoculation. In particular, miR156, miR159 and miR166 were greatly upregulated by TR4 inoculation. Because Cavendish banana is resistant to R1 and susceptible to TR4, it is possible that these differentially expressed miRNAs has facilitated the infection of TR4. On the other hand, this could also be a defence mechanism of the host to limit or/and delay the damage caused by the virulent Foc race.

Among the very few studies on small RNAs in pathogen inoculated banana, a study on Banana streak Mysore virus (BSMyV)-infected banana also reported upregulation of miR166 during viral infection (Sheeba et al. 2013). In a range of studies miR156 has been shown to be a critical regulator of phase change of plant growth by targeting the SQUAMOSA PROMOTER BINDING PROTEIN-LIKE (SPL) genes (Wu and Poethig 2006; Chuck et al. 2007; Wu et al. 2009; Wang et al. 2011). In Arabidopsis, miR156 was reported to be up-regulated by virus infection (Kasschau et al. 2003) as well as in tobacco (Bazzini et al. 2007), though down-regulation of miR156 was also revealed in response to the infection of Cronartium quercuum in pines (Lu et al. 2007) and powdery mildew in wheat (Xin et al. 2010). Cui et al. (2014) disclosed that the induction of miR156 improved adaptation and tolerance to environmental stresses including salt and drought stress by delaying the phase change of the plant. This could also be a possible explanation for the up-regulation of miR156 in this study. Both miR159 and miR166 target the transcription factors from the MYB and HD-Zip families respectively. Both of these gene families are involved in abscisic acid (ABA) signaling pathways which has linkage with plant defence pathways. It was previously speculated that a series of miRNAs including miR159 and miR166 are negative regulators of the abscisic acid (ABA) signaling pathways and subsequently positive regulator of the salicylic acid (SA)-mediated defence pathways (de Torres-Zabala et al. 2007; Zhang et al. 2011). In this scenario, it appears the up-regulation of miR159 and miR166 may be a mechanism to activate the SA-mediated defence pathway to facilitate host immunity upon TR4 infection. Considering that SA primarily mediates resistance to biotrophic and hemibiotrophic pathogens rather than necrothophs (Mengiste 2012), and that Fusarium oxysporum is considered as a hemibiotroph or a necrothoph (Thatcher et al. 2009; Ploetz 2015a; Dita et al. 2018), we think this speculation is still premature at this stage. More recently, miR159 and miR166 of cotton were found to be exported into $V$. dahliae and reduce the virulence during the host-pathogen interaction (Zhang et al. 2016). Considering $F$. oxysporum and $V$. dahliae are both fungal pathogens infecting plant vascular tissues, it is possible that the increased miR159 and miR166 in this study could also act as effectors and travel to $F$. oxysporum through an unknown defence pathway against aggressive Fusarium strains, although this requires further investigation.

In addition to miRNAs, we also found several $21 \mathrm{nt}$ and $24 \mathrm{nt}$ sRNAs that mapped to particular antisense loci of some banana genes and with different mapped read abundance between TR4-inoculated samples and control samples. It is possible that they are banana endogenous siRNAs involved in the plant defence mechanisms in response to the Foc infection, although further investigation is needed to confirm their derivation and function.

\section{Conclusions}

This study carried out the global small RNA profiling of Cavendish banana roots inoculated with two Foc races with different virulence upon banana plants. Our findings facilitate the understanding of the small RNA regulatory mechanisms during the banana-Foc interaction, which are useful resources for developing RNAi-based approaches to increase banana tolerance against aggressive $F o c$ races. 


\section{Methods}

\section{Plant materials and fungal culture}

Tissue culture plantlets of Cavendish cv. 'Williams' were initially transplanted into University of California (UC) soil mix (Baker 1957) and grown in a misted shade house for approximately 4 weeks before being transferred to a glasshouse for an additional 8 weeks until the plantlets had a pseudostem height of approximately $15 \mathrm{~cm}$.

Monoconidial cultures of accessions of Foc, BRIP 62880 (VCG 01213/16) and BRIP42161 (VCG 0124) were regenerated from lyophilized mycelia stored at $-80^{\circ} \mathrm{C}$ onto $1 / 2$ strength potato dextrose agar (PDA, Difco Laboratories) from the Northern Territory Department of Primary Industries and Brisbane Herbarium collection (Department of Agriculture and Forestry, Dutton Park, Australia), respectively. The cultures were incubated in dark conditions for 10 days at $24{ }^{\circ} \mathrm{C}$.

\section{Inoculation and sample collection}

Plants were inoculated using the Millet carrier method (Smith et al. 2008), where every $50 \mathrm{~g}$ sterile Japanese millet was inoculated with one $5 \times 5 \mathrm{~mm}^{2}$ mycelial plug of Foc. Due to quarantine restrictions on the handling of TR4 in Australia, inoculation of plants with TR4 BRIP 62880 was conducted at the Coastal Plains Research Station, Northern Territory (NT), Australia, whilst R1 BRIP 42161 was conducted at the University of Queensland, Brisbane, Queensland, Australia. Two mock-inoculated groups were operated in NT and Brisbane, respectively, to eliminate any influence resulting from the location difference. Plants were transferred into $40 \mathrm{~mm}$ ANOVA pots containing a bottom layer of $150 \mathrm{~g}$ of soil mix topped with $50 \mathrm{~g}$ of millet and $150 \mathrm{~g}$ of soil mix on top of that. Plants were transferred into these pots and filled with the soil mix. Plants were grown in the green house for the remainder of the trial. Control plants were inoculated using the same method described above but using uninoculated millet. Plant material was harvested at 28 days post-inoculation. Roots of the plants were harvested by removing excess soil, flash frozen and stored at $-80^{\circ} \mathrm{C}$ for RNA extractions. Three biological replicates consisting of five plants per replicate were used for each treatment group. For each biological replicate, root tissue was pooled prior to RNA extractions.

\section{RNA extraction and deep sequencing}

Pooled root tissue was snap frozen in liquid nitrogen and homogenized with a mortar and pestle. Total RNA was extracted using the EpiBio MasterPure Plant RNA Purification kit (Epibio Inc.) using the manufacturer's protocol. Small RNA library construction was conducted by the Australian Genome Research Facility (AGRF) using the Truseq Small RNA Library Preparation Kit (Illumina, California, USA). Sequencing (50 bp, single-end) was then conducted using the Illumina HiSeq2500 platform by AGRF.

\section{Bioinformatic analysis}

The FASTX-Toolkit package (http://hannonlab.cshl.edu/ fastx_toolkit/) (Gordon and Hannon 2010) was used to remove the adapter sequences from $50 \mathrm{bp}$ single-end reads. Only reads with lengths between 18 and $32 \mathrm{nt}$ were retained for further analysis. The SCRAM software package (Fletcher et al. 2018) was used to align processed reads to reference sequences and compare alignments of different treatments, with aligned reads normalized as reads aligned per million reads. The genome sequence of $M u s a$ acuminata (Musa) DH-Pahang (accessed at http://banana-genome-hub.southgreen.fr/) (D'Hont et al. 2012; Martin et al. 2016) was used as the banana reference. The genome assemblies Foc1_1.0 and FO_II5_V1 (accessed at http://fungi.ensembl.org/index.html) (Guo et al. 2014) were used as Foc R1 and Foc TR4 references respectively.

\section{miRNA identification and target gene prediction}

The MiRDeep2 software package (Friedländer et al. 2012) was used to identify known and novel miRNAs. Known miRNAs were determined by aligning sequencing reads to published mature miRNA and miRNA precursor sequences available in miRBase (Release 21, http://www.mirbase.org/) (Griffiths-Jones et al. 2008), allowing zero mismatches in the first 18 nucleotides and up to two mismatches thereafter. Reads that were not mapped to miRNAs from miRBase were aligned to the banana genome (D'Hont et al. 2012; Martin et al. 2016). Those with significant randfold $P$-value $(<0.05)$ in the MiRDeep2 pipeline were considered as putative novel miRNAs.

Target genes of identified miRNAs were predicted using the psRNATarget program (https://plantgrn.noble.org/ psRNATarget/) (Dai and Zhao 2011; Dai et al. 2018). Version 1 of all gene coding sequences of Musa acuminata (banana) released from the Banana Genome Hub was used as the target transcript library. The default settings of Scoring Scheme V2 was put as predicting criteria. The predicted target genes of identified known miRNAs and putative novel miRNAs were then submitted to the Blast2Go program (http://www.blast2go.com) (Conesa et al. 2005) for gene ontology (GO) analysis.

\section{qRT-PCR validation of differentially expressed miRNAs} and their predicted targets

Total RNA extracted for deep sequencing was also used for qPCR analysis. RNA samples were treated with Invitrogen's Turbo RNase-free DNase I (Invitrogen, California, USA) to eliminate any genomic DNA remaining in the samples. A $500 \mathrm{ng}$ quantity of total RNA was used to synthesize cDNA of miRNAs using the miScript Plant RT Kit (QIAGEN, Limberg, Netherlands). The following 
conditions were used for reverse transcription: $37^{\circ} \mathrm{C}$ for $2 \mathrm{~h}, 95^{\circ} \mathrm{C}$ for $5 \mathrm{~min}$ with a final hold step at $4{ }^{\circ} \mathrm{C}$. Quantitative real time PCR was performed with Rotor-Gene Q platform (QIAGEN, Limberg, Netherlands) in a $20 \mu \mathrm{L}$ reaction mixture, which consisted of $2 \mu \mathrm{L}$ diluted cDNA, $2 \mu \mathrm{L}$ of $10 \times$ miScript universal forward primer, $2 \mu \mathrm{L}$ of $8 \mu \mathrm{M}$ reverse primer, $10 \mu \mathrm{L}$ of $2 \times$ QuantiTect SYBR Green PCR Master Mix (QIAGEN, Limberg, Netherlands) and $4 \mu \mathrm{L}$ RNase-free water. The following conditions were used for PCR amplification: $95^{\circ} \mathrm{C}$ for $15 \mathrm{~min}$, and then 40 cycles of $94{ }^{\circ} \mathrm{C}$ for $15 \mathrm{~s}, 55^{\circ} \mathrm{C}$ for $30 \mathrm{~s}$ and $70^{\circ} \mathrm{C}$ for $30 \mathrm{~s}$. To validate the expression of predicted target genes, $1 \mu \mathrm{g}$ of total RNA was used to synthesize cDNA using SensiFAST cDNA Synthesis Kit (Bioline, London, UK). Conditions for reverse transcription were as follows: $25^{\circ} \mathrm{C}$ for $10 \mathrm{~min}, 42^{\circ} \mathrm{C}$ for $15 \mathrm{~min}, 85^{\circ} \mathrm{C}$ for $5 \mathrm{~min}$ and hold at $4{ }^{\circ} \mathrm{C}$. The $20 \mu \mathrm{L}$ reaction mixture for qRT-PCR consisted of $2 \mu \mathrm{L}$ of diluted cDNA, $1 \mu \mathrm{L}$ of $10 \mu \mathrm{M}$ forward primer, $1 \mu \mathrm{L}$ of $10 \mu \mathrm{M}$ reverse primer, $10 \mu \mathrm{L}$ of $2 \times$ SensiFAST SYBR ${ }^{\circ}$ No-ROX Mix (Bioline, London, $\mathrm{UK}$ ) and $6 \mu \mathrm{L}$ RNase-free water. The reaction was performed using the Rotor-Gene Q platform (QIAGEN, Limberg, Netherlands) following the conditions below: $95^{\circ} \mathrm{C}$ for $2 \mathrm{~min}$, then 40 cycles of $95^{\circ} \mathrm{C}$ for $5 \mathrm{~s}$, $60{ }^{\circ} \mathrm{C}$ for $15 \mathrm{~s}$ and $72{ }^{\circ} \mathrm{C}$ for $10 \mathrm{~s}$.

A melt-curve was generated in the Rotor-Gene Q Series Software to analyze the specificity of the miRNA and target gene PCR products. Data obtained from this software was imported to the LinRegPCR Software (University of Amsterdam, The Netherlands) to determine the primer efficiency. $\mathrm{Ct}$ is the cycle threshold of the PCR reaction. Primer efficiency was averaged from all reactions using the same primer. $\mathrm{Ct}$ value was averaged from two technical replicates. miR399 was used as an endogenous reference for miRNA expression analysis. L2 was used as an endogenous reference gene for target gene expression analysis. Relative expression of miRNAs and target genes was calculated using the following formula:

Relative Expression $(\mathrm{RE})=\mathrm{PE}_{\text {target }}{ }^{(-\mathrm{Ct} \text { target })} / \mathrm{PE}_{\text {reference }}{ }^{(-\mathrm{Ct} \text { reference })}$

The RE of each treatment was then compared. One-way analysis of variation (ANOVA) was used to determine the significance of differences. Primers were designed using Primer 3.0 plus online system listed in Additional file 4: Table S4.

\section{Additional files}

Additional file 1: Figure S1. Fold change of miRNA expression in inoculated groups as compared to control groups. miR156a, miR159f and miR166c showed the most induction of expression in TR4_I as compared to TR4_C. $X$ axis represents $\log _{2}$ (Mean of TR4_l/Mean of TR4_C) or $\log 2$ (Mean of R1_l/Mean of R1_C). Figure S2. sRNA mapping profile of
Ma02 g06960. The $X$ axis represents the full length of the gene sequence of Ma02_g06960. The $Y$ axis represents the mapped read abundance when aligning sequenced reads to the gene sequence. Above zero means that the reads mapped to the sense strand, while below zero means that the reads mapped to the antisense strand. Green color represents $21 \mathrm{nt}$ reads. Blue color represents $24 \mathrm{nt}$ reads. The abundance of mapped reads was induced in TR4_I and R1_I as compared to TR4_C and R1_C, respectively. (DOCX $261 \mathrm{~kb}$ )

Additional file 2: Table S1. Putative novel miRNAs predicted using the miRDeep2 package (DOCX $17 \mathrm{~kb}$ )

Additional file 3: Table S2. Target gene prediction for (a) identified known miRNAs and (b) putative novel miRNAs. (XLSX 35 kb)

Additional file 4: Table S3. $21 \mathrm{nt}$ and $24 \mathrm{nt}$ read count of each treatment group that mapped to the listed banana genes. Table S4.

Primers for qRT-PCR validation of the expression of selected miRNAs and their target genes. (DOCX $24 \mathrm{~kb})$

\section{Abbreviations}

ANOVA: Analysis of variation; DCL: Dicer-like; Foc: Fusarium oxysporum f. sp. cubense; miRNA: MicroRNA; NGS: Next Generation Sequencing; PTGS: Posttranscriptional silencing; R1: Race 1; RISC: RNA-induced silencing complex; RPMR: Reads per million reads; siRNA: Small interfering RNA; sRNA: Small RNA; TGS: Transcriptional silencing; TR4: Tropical race 4

\section{Acknowledgements}

Not applicable.

\section{Authors' contributions}

S Fei and NM conceived and designed the experiment. EC conducted the plant inoculation and RNA extractions. S Fei conducted the qRT-PCR validation and statistics analysis on experimental data. S Fletcher processed the sequenced small RNA raw data. S Fletcher and S Fei performed all the bioinformatics analyses. JP, JB, EA and NM provided technical support and edited the manuscript. All authors read and approved the final manuscript.

\section{Funding}

This study was funded by the Accelerated Partnership Grant, Queensland Government (2014000652). Shulang Fei's PhD programme is supported by the China Scholarship Council and the University of Queensland.

\section{Availability of data and materials}

The sequencing data are submitted to NCBI Sequence Read Archive (SRA) and are pending approval. All data analyzed in this study are included in this published article and the additional files.

Ethics approval and consent to participate

Not applicable.

\section{Consent for publication}

Not applicable.

\section{Competing interests}

The authors declare that they have no competing interests.

\section{Author details}

${ }^{1}$ Queensland Alliance for Agriculture and Food Innovation, The University of Queensland, St Lucia, QLD 4072, Australia. ${ }^{2}$ School of Agriculture and Food Sciences, The University of Queensland, St Lucia, QLD 4072, Australia. ${ }^{3}$ School of Biological Sciences, University of Western Australia, Crawley, Western Australia 6009, Australia.

Received: 1 February 2019 Accepted: 3 June 2019

Published online: 25 June 2019

\section{References}

Axtell MJ. Classification and comparison of small RNAs from plants. Annu Rev Plant Biol. 2013;64:137-59.

Baker KF. The U.C. system for producing healthy container-grown plants through the use of clean soil, clean stock, and sanitation. Berkeley: University of 
California, Division of Agricultural Sciences, Agricultural Experiment Station, Extension Service; 1957.

Bazzini AA, Hopp HE, Beachy RN, Asurmendi S. Infection and coaccumulation of tobacco mosaic virus proteins alter microRNA levels, correlating with symptom and plant development. Proc Natl Acad Sci U S A. 2007;104:12157.

Bonnet $E$, Wuyts J, Rouzé $P$, Van de Peer $Y$. Evidence that microRNA precursors, unlike other non-coding RNAs, have lower folding free energies than random sequences. Bioinformatics. 2004;20:2911-7.

Castel SE, Martienssen RA. RNA interference (RNAi) in the nucleus: roles for small RNA in transcription, epigenetics and beyond. Nat Rev Genet. 2013;14:100-12.

Chai J, Feng R, Shi H, Ren M, Zhang Y, Wang J. Bioinformatic identification and expression analysis of banana microRNAs and their targets. PLoS One. 2015; 10:e0123083.

Chen D, Meng Y, Yuan C, Bai L, Huang D, Lv S, et al. Plant siRNAs from introns mediate DNA methylation of host genes. RNA. 2011;17:1012-24.

Chen L, Luan Y, Zhai J. Sp-miR396a-5p acts as a stress-responsive genes regulator by conferring tolerance to abiotic stresses and susceptibility to Phytophthora nicotianae infection in transgenic tobacco. Plant Cell Rep. 2015;34:2013-25.

Chuck G, Cigan AM, Saeteurn K, Hake S. The heterochronic maize mutant Corngrass 1 results from overexpression of a tandem microRNA. Nat Genet. 2007;39:544.

Conesa A, Gotz S, Garcia-Gomez JM, Terol J, Talon M, Robles M. Blast2GO: a universal tool for annotation, visualization and analysis in functional genomics research. Bioinformatics. 2005;21:3674-6.

Cui LG, Shan JX, Shi M, Gao JP, Lin HX. The miR156-SPL9-DFR pathway coordinates the relationship between development and abiotic stress tolerance in plants. Plant J. 2014;80:1108-17.

Dai X, Zhao PX. psRNATarget: a plant small RNA target analysis server. Nucleic Acids Res. 2011;39:W155-9.

Dai X, Zhuang Z, Zhao PX. psRNATarget: a plant small RNA target analysis server (2017 release). Nucleic Acids Res. 2018;46:W49-w54.

Dale J, James A, Paul J-Y, Khanna H, Smith M, Peraza-Echeverria S, et al. Transgenic cavendish bananas with resistance to Fusarium wilt tropical race 4. Nat Commun. 2017;8:1496.

Davey MW, Gudimella R, Harikrishna JA, Sin LW, Khalid N, Keulemans J. A draft Musa balbisiana genome sequence for molecular genetics in polyploid, interand intra-specific Musa hybrids. BMC Genomics. 2013;14:683.

de Torres-Zabala M, Truman W, Bennett MH, Lafforgue G, Mansfield JW, Rodriguez Egea P, et al. Pseudomonas syringae pv. Tomato hijacks the Arabidopsis abscisic acid signalling pathway to cause disease. EMBO J. 2007;26:1434-43.

D'Hont A, Denoeud F, Aury JM, Baurens FC, Carreel F, Garsmeur O, et al. The banana (Musa acuminata) genome and the evolution of monocotyledonous plants. Nature. 2012;488:213-7.

Dita M, Barquero M, Heck D, Mizubuti ESG, Staver CP. Fusarium wilt of banana: current knowledge on epidemiology and research needs toward sustainable disease management. Front Plant Sci. 2018;9:1468.

Fahlgren N, Howell MD, Kasschau KD, Chapman EJ, Sullivan CM, Cumbie JS, et al. High-throughput sequencing of Arabidopsis microRNAs: evidence for frequent birth and death of MIRNA genes. PLoS One. 2007;2:e219.

Fletcher SJ, Boden M, Mitter N, Carroll BJ. SCRAM: a pipeline for fast index-free small RNA read alignment and visualization. Bioinformatics. 2018;34:2670-2.

Friedländer MR, Mackowiak SD, Li N, Chen W, Rajewsky N. miRDeep2 accurately identifies known and hundreds of novel microRNA genes in seven animal clades. Nucleic Acids Res. 2012;40:37-52.

Ghag SB, Shekhawat UK, Ganapathi TR. Small RNA profiling of two important cultivars of banana and overexpression of miRNA156 in transgenic banana plants. PLoS One. 2015;10:e0127179.

Gordon A, Hannon G. Fastx-toolkit. FASTQ/A short-reads pre-processing tools. 2010.https://hannonlabcshledu/fastx_toolkit. Accessed 10 Jan 2017.

Griffiths-Jones S, Saini HK, van Dongen S, Enright AJ. miRBase: tools for microRNA genomics. Nucleic Acids Res. 2008;36(Database issue):D154-8.

Guo L, Han L, Yang L, Zeng H, Fan D, Zhu Y, et al. Genome and transcriptome analysis of the fungal pathogen Fusarium oxysporum f. sp. cubense causing banana vascular wilt disease. PLoS One. 2014;9:e95543.

Gupta OP, Sharma P, Gupta RK, Sharma I. Current status on role of miRNAs during plant-fungus interaction. Physiol Mol Plant Pathol. 2014;85:1-7.

Hwang SC, Ko WH. Cavendish banana cultivars resistant to Fusarium wilt acquired through somaclonal variation in Taiwan. Plant Dis. 2004;88:580-8.

Islam W, Islam SU, Qasim M, Wang L. Host-pathogen interactions modulated by small RNAs. RNA Biol. 2017;14:891-904.
Kamthan A, Chaudhuri A, Kamthan M, Datta A. Small RNAs in plants: recent development and application for crop improvement. Front Plant Sci. 2015;6:208.

Kasschau KD, Xie Z, Allen E, Llave C, Chapman EJ, Krizan KA, et al. P1/HC-pro, a viral suppressor of RNA silencing, interferes with Arabidopsis development and miRNA function. Dev Cell. 2003:4:205-17.

Lee WS, Gudimella R, Wong GR, Tammi MT, Khalid N, Harikrishna JA. Transcripts and MicroRNAs responding to salt stress in Musa acuminata Colla (AAA Group) cv. Berangan roots. PLoS One. 2015;10:e0127526.

Lu S, Sun YH, Amerson H, Chiang VL. MicroRNAs in loblolly pine (Pinus taeda L.) and their association with fusiform rust gall development. Plant J. 2007;51: 1077-98.

Martin G, Baurens F-C, Droc G, Rouard M, Cenci A, Kilian A, et al. Improvement of the banana "Musa acuminata" reference sequence using NGS data and semiautomated bioinformatics methods. BMC Genomics. 2016;17:243.

Mengiste T. Plant immunity to necrotrophs. Annu Rev Phytopathol. 2012;50:267-94.

Muthusamy M, Uma S, Backiyarani S, Saraswathi MS. Computational prediction, identification, and expression profiling of microRNAs in banana (Musa spp.) during soil moisture deficit stress. J Hortic Sci Biotechnol. 2015;89:208-14.

Navarro L, Dunoyer P, Jay F, Arnold B, Dharmasiri N, Estelle M, et al. A plant miRNA contributes to antibacterial resistance by repressing auxin signaling. Science. 2006;312:436.

Ouyang S, Park G, Atamian HS, Han CS, Stajich JE, Kaloshian I, et al. MicroRNAs suppress NB domain genes in tomato that confer resistance to Fusarium oxysporum. PLoS Pathog. 2014;10:e1004464.

Pietro AD, Madrid MP, Caracuel Z, Delgado-Jarana J, Roncero MI. Fusarium oxysporum: exploring the molecular arsenal of a vascular wilt fungus. Mol Plant Pathol. 2003;4:315-25.

Ploetz RC. Fusarium wilt of banana is caused by several pathogens referred to as Fusarium oxysporum f. sp. cubense. Phytopathology. 2006;96:653-6.

Ploetz RC. Fusarium wilt of Banana. Phytopathology. 2015a;105:1512-21.

Ploetz RC. Management of Fusarium wilt of banana: a review with special reference to tropical race 4. Crop Prot. 2015b;73:7-15.

Podevin N, Krauss A, Henry I, Swennen R, Remy S. Selection and validation of reference genes for quantitative RT-PCR expression studies of the non-model crop Musa. Mol Breed. 2012;30:1237-52.

Ruiz-Ferrer V, Voinnet O. Roles of plant small RNAs in biotic stress responses. Annu Rev Plant Biol. 2009;60:485-510.

Sheeba MM, Selvarajan R, Mustaffa MM. Prediction and identification of microRNA from banana infected with BSMYV. Madras Agric J. 2013;100:513-8

Smith L, Smith MK, Tree D, O'Keefe D, Galea VJ. Development of a small-plant bioassay to assess banana grown from tissue culture for consistent infection by Fusarium oxysporum f. sp. cubense. Australas Plant Pathol. 2008;37:171-9.

Song S, Chen X, Huang D, Xu Y, Zeng H, Hu X, et al. Identification of miRNAs differentially expressed in Fusarium wilt-resistant and susceptible banana varieties. S Afr J Bot. 2016;106:244-9.

Song S, Huang D, Xu Y, Guo G, Li Y, Jin Z. A study on banana miRNA and its application in resistance to Fusarium wilt. J Plant Breed Crop Sci. 2015;7:307-9.

Song S, Xu Y, Huang D, Ashraf MA, Li J, Hu W, et al. Identification and characterization of miRNA169 family members in banana (Musa acuminata L. ) that respond to Fusarium oxysporum f. sp. cubense infection in banana cultivars. PeerJ. 2018;6:e6209.

Thatcher LF, Manners JM, Kazan K. Fusarium oxysporum hijacks COI1-mediated jasmonate signaling to promote disease development in Arabidopsis. Plant J. 2009;58:927-39.

Vaucheret H. Post-transcriptional small RNA pathways in plants: mechanisms and regulations. Genes Dev. 2006;20:759-71.

Wang JW, Park MY, Wang L, Koo Y, Chen XY, Weigel D, et al. MiRNA control of vegetative phase change in trees. PLoS Genet. 2011;7:e1002012.

Wu G, Park MY, Conway SR, Wang JW, Weigel D, Poethig RS. The sequential action of miR156 and miR172 regulates developmental timing in Arabidopsis. Cell. 2009;138:750-9.

Wu G, Poethig RS. Temporal regulation of shoot development in Arabidopsis thaliana by miR156 and its target SPL3. Development. 2006;133:3539.

Xin M, Wang Y, Yao Y, Xie C, Peng H, Ni Z, et al. Diverse set of microRNAs are responsive to powdery mildew infection and heat stress in wheat (Triticum aestivum L.). BMC Plant Biol. 2010;10:123.

Zhang T, Zhao YL, Zhao JH, Wang S, Jin Y, Chen ZQ, et al. Cotton plants export microRNAs to inhibit virulence gene expression in a fungal pathogen. Nat Plants. 2016;2:16153. 
Zhang W, Gao S, Zhou X, Chellappan P, Chen Z, Zhou X, et al. Bacteriaresponsive microRNAs regulate plant innate immunity by modulating plant hormone networks. Plant Mol Biol. 2011;75:93-105.

Ready to submit your research? Choose BMC and benefit from:

- fast, convenient online submission

- thorough peer review by experienced researchers in your field

- rapid publication on acceptance

- support for research data, including large and complex data types

- gold Open Access which fosters wider collaboration and increased citations

- maximum visibility for your research: over $100 \mathrm{M}$ website views per year

At $B M C$, research is always in progress.

Learn more biomedcentral.com/submissions 